Classification

Physics Abstracts

61.16Ch $-68.55 \mathrm{Ln}-85.40 \mathrm{Vb}-68.18+\mathrm{p}$

\title{
Scanning surface harmonic microscopy: Application to silicon and Langmuir-Blodgett films on silicon
}

\author{
Jean-Philippe Bourgoin $\left(^{*}\right)$, Matthew Bruce Johnson and Bruno Michel \\ IBM Research Division, Zurich Research Laboratory, Säumerstrasse 4, 8803 Rüschilikon, \\ Switzerland
}

(Received July 4; accepted December 20, 1994)

\begin{abstract}
The scanning surface harmonic microscope is a local probe method in which a microwave signal is applied across a tip-sample tunneling gap generating higher harmenics, which can then be detected. Thus this microscope is sensitive to nonlinearities in the tip-sample junction. In $\mathrm{Si}$ and other semiconductors it is sensitive to nonlinearities in the tip-sample capacitance voltage characteristics $(C / V)$. Such a local $C / V$ has been used to delineate regions of different dopant type and concentration in $\mathrm{n}^{+} / \mathrm{p}$ silicon gratings. On Langmuir-Blodgett films of dielectrics, such as docosanoic acid on $\mathrm{Si}$, the scanning surface harmonic microscope is sensitive to changes in film thickness which cause shifts in the $C / V$ characteristics of the underlying $\mathrm{Si}$.
\end{abstract}

\section{Introduction.}

The use of high-frequency modulation of bias voltage has recently opened up new possibilities for the scanning tunneling microscope (STM), including investigations of insulating surfaces and studies of the nonlinear properties of the surface region [1-4]. In a so-called scanning surface harmonic microscope (SSHM), or alternating current scanning tunneling microscope (ACSTM), an STM is situated within a microwave cavity, where an ac microwave bias voltage (typically 1 $\mathrm{GHz}$ ) is superimposed on the usual dc bias voltage. As a consequence of nonlinearities present in the tip-sample junction, higher harmonics of the ac voltage are generated and can be detected. These higher harmonics provide local information on the nonlinearities from which they originate. Futhermore, by using higher harmonic feedback, no dc tunneling current is necessary, so insulating surfaces can be investigated.

The tip-sample junction can be modelled as a lumped $R C$ circuit. The mechanisms responsible for higher harmonics generation are associated with nonlinearities in either $R$ or $C$ or both. Nonlinearities involving the tip-sample resistance $R$ include Coulomb blockade [1], variation of local

$\left({ }^{*}\right)$ On leave from: Service de Chimie Moleculaire, Commissariat à l'Energie Atomique, C.E. Saclay, 91191 Gif/Yvette Cedex, France. 
densities of states [1-3], and voltage-dependent elastic deformation [3]. Nonlinearities involving the tip-sample capacitance $C$ include voltage-dependent elastic deformation [2, 3], voltagedependent polarization within the tunneling gap [1], e.g. water in the gap, and a voltage-dependent depletion region for semiconductors [3]. The nonlinear generation mechanism has been clearly identified in two cases: graphite [3,5] and $\mathrm{WSe}_{2}[3]$. In the former, the higher harmonics are generated due to the nonlinearity of the dc $I / V$ curve. This results both from the variation of the local density of states with voltage and from voltage-dependent elastic deformation of the tip-sample gap. In the latter, the higher harmonics are due to the nonlinearity of the $C(V)$ curve.

We have studied silicon surfaces of various $n$ and p-type concentrations as well as LangmuirBlodgett (LB) films deposited on silicon for the following purposes: i) to identify the mechanism of higher harmonic generation in silicon, ii) to gain knowledge about SSHM investigations of dielectrics on silicon, and iii) to understand the origin of the contrast in SSHM images of LB films. In this paper, we present the results on medium-doped p-type silicon, $(10-\mu \mathrm{m}) \mathrm{n}^{+} / \mathrm{p}$ silicon gratings, and LB films of docosanoic acid (behenic acid) deposited on silicon substrates. We show that higher harmonics signals are generated in silicon due to the nonlinearity of the $C(V)$ characteristics, and that an SSHM can thus be used for dopant profiling of semiconductors. We also show that the contrast observed in constant higher harmonics images of LB films is due to the modulation of the higher harmonics signal generated in the underlying silicon substrate.

\section{Experimental details.}

The SSHM consists of an STM operated in a microwave cavity. The experimental setup, shematically shown in figure 1 , is the same as that in [2] with the addition of a wet/dry nitrogen flow. This modification allows the relative humidity of the cavity to be varied between $1-75 \%$. The cavity resonance frequency $\left(f_{\mathrm{c}}\right)$ is $2.7 \mathrm{GHz}$, so that when the input signal is $f_{\mathrm{c}}, f_{\mathrm{c}} / 2$ or $f_{\mathrm{c}} / 3$, the fundamental, second or third harmonic (SH or TH) signals are detected, respectively. p-type Si(100) samples (boron-doped $\left.2 \times 10^{18} \mathrm{~cm}^{-3}\right)$ and $(10 \mu \mathrm{m}) \mathrm{n}^{+} / \mathrm{p}$ silicon gratings, prepared by implanting phosphorus ions in p-type silicon $\left[(100) 1 \times 10^{15} \mathrm{~cm}^{-3}\right]$, were studied. These samples were cleaned using standard procedures [6,7], but with the final steps modified to be a diluted HF dip followed by the growth of a 2-nm-thick chemical oxide using a 5:1:1 $\mathrm{H}_{2} \mathrm{O}: \mathrm{H}_{2} \mathrm{O}_{2}: \mathrm{HCl}$ solution and a rinse in HPLC-grade water. The typical tunneling conditions were: $\mathrm{W}$ tip with $30-\mathrm{nm}$ tip radius; dc conditions: tunnel current $20 \mathrm{pA}$ and tip voltage $-2 \mathrm{~V}$ (tip-sample separation about $3 \mathrm{~nm}$ ); and microwave input power $2 \mathrm{dBm}$ (1.6 V peak-to-peak across the gap). Approximately $99 \%$ of the

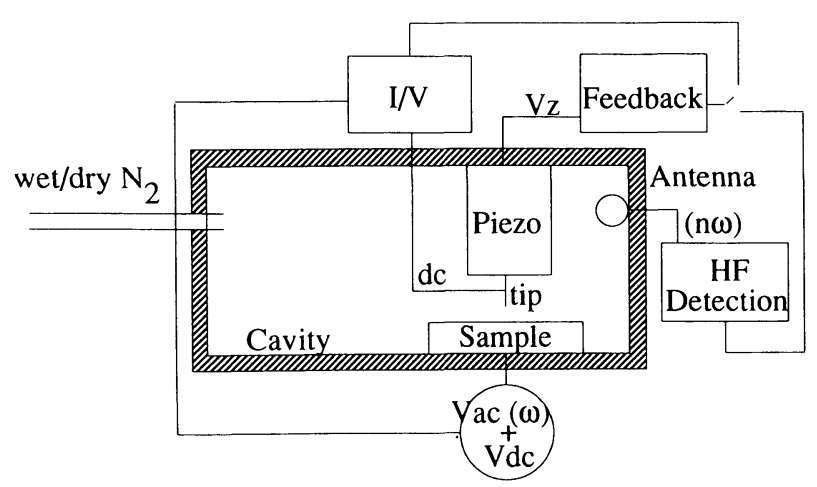

Fig. 1. - Experimental setup. 
ac power is reflected at the high impedance tip-sample junction. Finally, LB films deposited on low-doped p-type silicon $\left[(100) 7 \times 10^{15} \mathrm{~cm}^{-3}\right.$ ] have been investigated [8]. These films consisted of twelve monomolecular layers of docosanoic acid deposited at a surface pressure of $35 \mathrm{mN} / \mathrm{m}$ on top of p-type silicon. These LB films have been investigated at a constant TH signal of $60 \mathrm{nV}$ (signal at the antenna), and a dc bias voltage of $-0.5 \mathrm{~V}$ with either $\mathrm{W}$ or Pt-Ir etched tips [9].

\section{Results and discussion.}

3.1 SiliCON SAMPLES. - The tip-sample junction can be modeled as a lumped $R C$ circuit, where the tip-sample capacitance $C$, results from the series association of the gap capacitance, the oxide capacitance, and the silicon capacitance as shown in figure 2. For the experimental conditions used here, the tip-sample capacitance dominates over the tip-sample resitance.

Thus, for an applied voltage of $V(t)=V+v e^{j \omega t}$, where $V$ is the dc bias voltage, and $v e^{j \omega t}$ the microwave input voltage, the output microwave signal is generated by $i=j \omega C(V(t)) v e^{j \omega t}$. Expanding this expression for small signals about $V$ yields

$$
i=j \omega\left(C(V) v e^{j \omega t}+C^{\prime}(V) v^{2} e^{2 j \omega t}+\frac{1}{2} C^{\prime \prime}(V) v^{3} e^{3 j \omega t}+\ldots\right) .
$$

Consequently, in agreement with the work of Seifert et al. on $\mathrm{WSe}_{2}$ [3], $\mathrm{SH}$ and $\mathrm{TH}$ signals should be proportional to the first and second derivatives of $C$ with respect to $V$, whereas the fundamental signal is directly proportional to $C$.

The capacitive tip-sample junction can be modeled as a metal-insulator-semiconductor (MIS) junction, in which the tip and tunneling gap, including the thin oxide, correspond to the metallic gate and the gate oxide, respectively. The MIS capacitance varies in a nonlinear fashion with voltage as shown in figure $3 \mathrm{a}$ for high and low-frequency regimes [10]. The change in $C$ common to both regimes (near flat band) corresponds to the change in the semiconductor from accumulation through depletion to inversion, as labeled. The change in $C$ observed only at low frequencies involves the change in minority carrier concentration, which is a slow process. The experimental capacitance data corresponding to the fundamental signal have been plotted in figure $3 \mathrm{~b}$. Comparing the experimentally measured capacitances in figure $3 \mathrm{~b}$ to their MIS-modeled counterparts, the general agreeement between the experimental and high-frequency models is good, as expected because SSHM is a microwave technique. Differences in the exact position of the maximum slope of the theoretical and experimental $C / V$ curves are due to the effects of surface states, traps, or oxide charges, as also observed in conventional $C / V$ measurements [10].

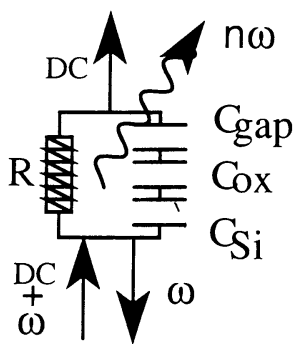

a)

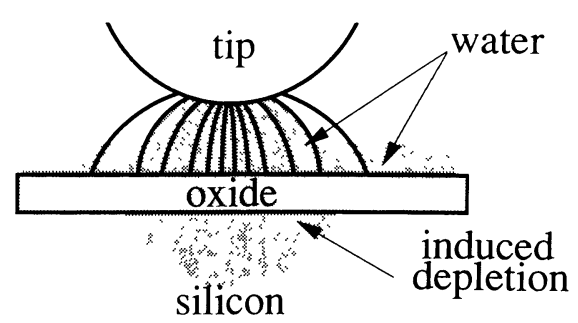

b)

Fig. 2. - Schematics of SSHM tip-sample region. a) Lumped RC model of tip-sample junction including the gap, oxide, and silicon capacitances. b) Tip-sample junction showing a "nano-droplet" of water. 


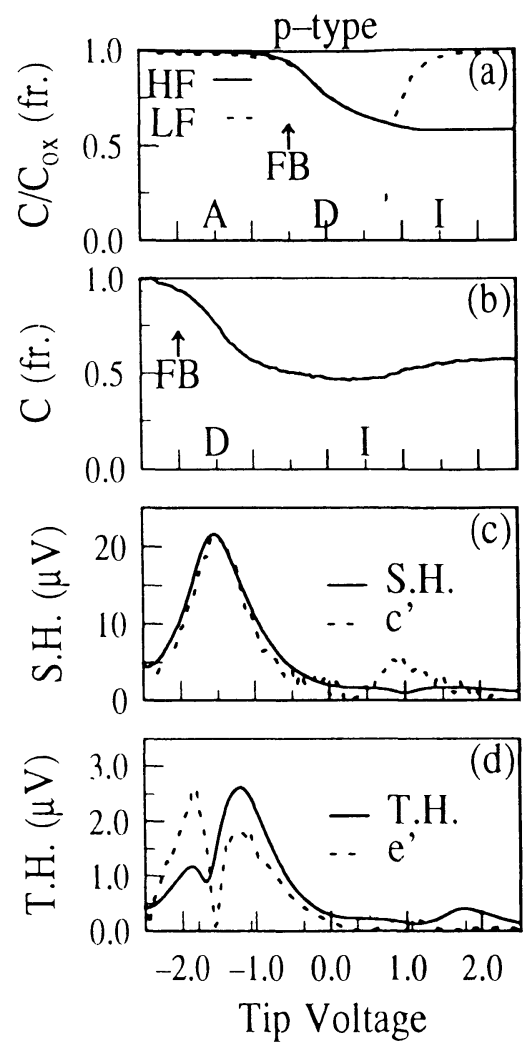

Fig. 3. - Calculated $C / C_{\text {ox }}$ versus bias voltage for p-type $\mathrm{SiO}_{2} / \mathrm{Si}$ samples. Both high-frequency (solid line) and low-frequency (dotted line) capacitances are shown. Plots (solid line) show b) fundamental, c) SH, and d) TH SSHM signals for p-type Si(100) samples, respectively. Tunneling conditions: tip voltage $-2 \mathrm{~V}$, current $20 \mathrm{pA}$, and $75 \%$ relative humidity. In the $\mathrm{SH}(\mathrm{TH})$ plots, the dotted scans are the derivatives of the smoothed fundamental $(\mathrm{SH})$ scans.

Comparing the curves in figure $3 \mathrm{~b}$ with those in figures $3 \mathrm{c}$ and $\mathrm{d}$, it is clear that $\mathrm{SH}$ and $\mathrm{TH}$ signals are indeed the absolute value [11] of the first and second voltage derivatives, respectively, of the capacitance. The agreement between peak positions is very good, aside from small differences in relative intensities because the measurements are taken at different frequencies, and at a probing ac voltage $v$ that is not really negligible compared to $V$, the dc bias voltage. Thus the large peak in the SH spectra (generally double peaks in $\mathrm{TH}$ ) correspond to the voltage at which the semiconductor is depleted. It should be noted that the data corresponding to the fundamental spectrum are measured by the variation of the cavity's resonance frequency. Thus, they are far noisier than $\mathrm{SH}$ and $\mathrm{TH}$ data, which contain essentially the same information. As a final justification of equation (1), the microwave amplitude dependences of the fundamental, $\mathrm{SH}$ and TH are indeed 1,2 , and 3 , respectively. The smaller feature observed at positive voltage probably originates from minority carrier generation from surface traps at the slightly pinned, inverted surface, which is consistent with simultaneously recorded $I / V$ spectra, and with other experimental observations [12]. Moreover, the feature is usually larger for low doping levels, it can be enhanced by illumination, and is dependent on surface contamination (cleaning conditions in particular).

It is important to note that the control of relative humidity is crucial in these experiments. 


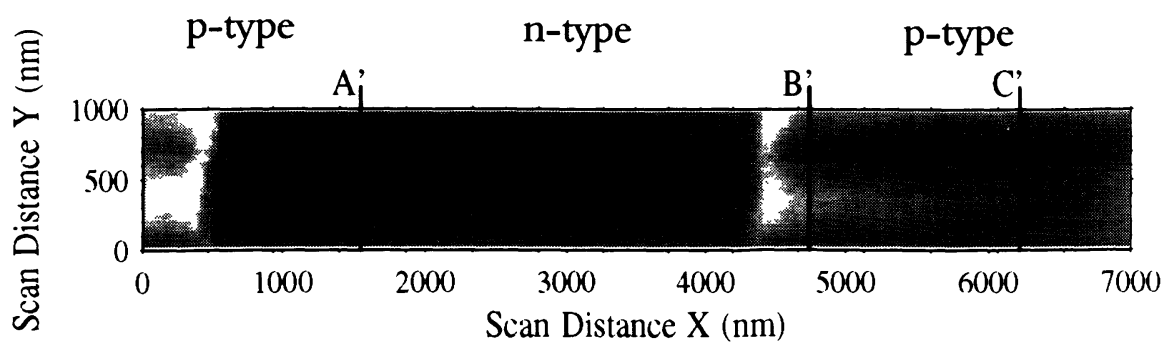

(a)

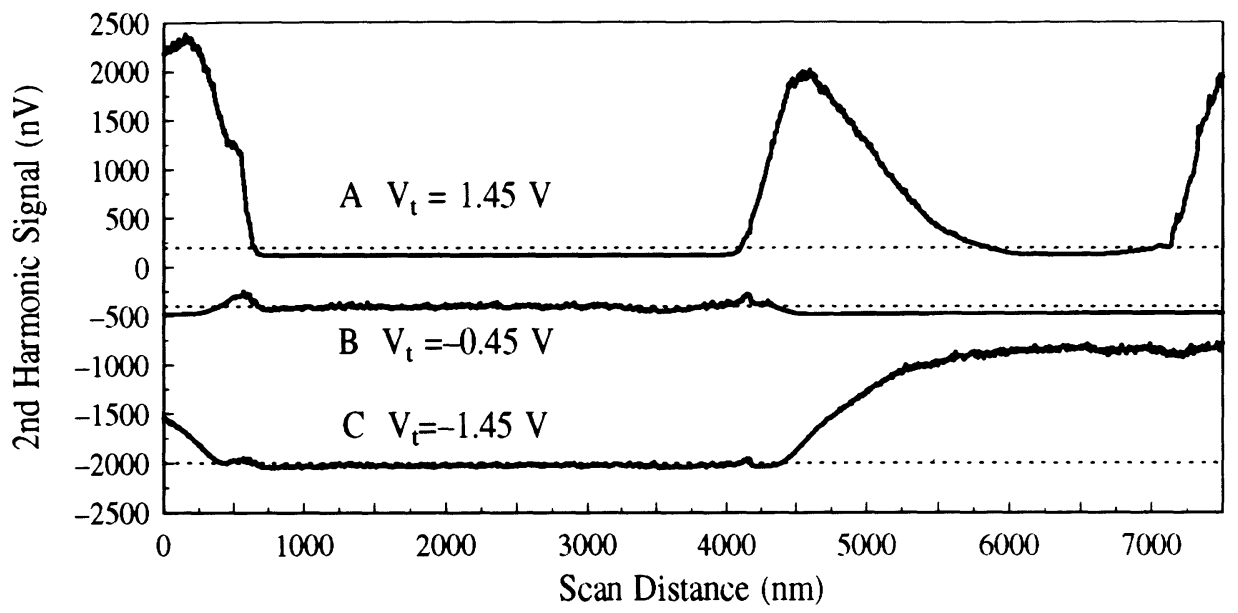

(b)

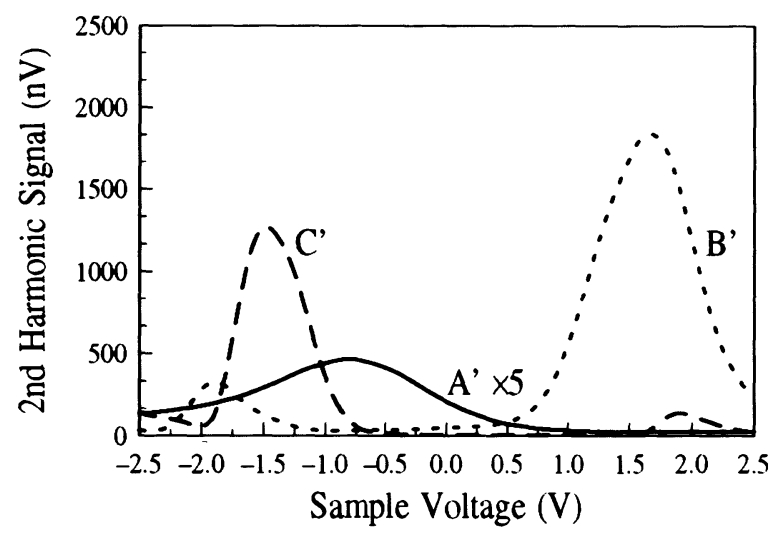

(c)

Fig. 4. - SSHM of a planar $\mathrm{n}^{+} / \mathrm{p}$ grating. a), b) Vertically aligned plots versus scan position perpendicular to the grating direction. a) Gray-scale representation image of $\mathrm{SH} v$ s. scan position. Same tunneling conditions as in figure 3. b) SH line scans $\mathrm{A}, \mathrm{B}$ and $\mathrm{C}$ for $-1.45,-0.45$, and $1.45 \mathrm{~V}$, respectively. For clarity, origins of the curve (dotted lines) have been shifted. c) SH spectra A', B', and C' taken at positions corresponding to lines A', B', and C' of a), respectively.

For the lowest relative humidity conditions (about 1\%), almost no higher harmonic signals are observed, whereas for high-humidity conditions, harmonic signals are detected with a typical $S / N$ value of 300 [13]. This is because water with $\epsilon_{\mathrm{r}}=80$ (even up to $1 \mathrm{GHz}$ ) greatly increases the tip-sample capacitance and thus the higher harmonic generation. In addition, the spatial extent 
of the water "nano-droplet" formed in the tunnel gap, as shown in figure 2a, should reduce the contribution of the fringe fields. Indeed, by varying the tip radius and relative humidity, we can reduce the size of the nano-droplet and hence improve the lateral resolution, albeit at the expense of the higher-harmonic signal.

As a consequence of its sensitivity to the local capacitance/voltage characteristics of semiconductor samples, an SSHM can be used as a dopant profiler [14]. As an example, figure 4 shows SSHM results on a $(10 \mu \mathrm{m}) \mathrm{n}^{+} / \mathrm{p}$ silicon grating. Parts (a) and (b) show properties across a period of the grating aligned vertically. Part (a) is a narrow second-harmonic scan taken at a tip voltage of $-2 \mathrm{~V}$ in the usual constant-current feedback mode with a dc current of $20 \mathrm{pA}$. Part (c) shows three representative spectra corresponding to the three regions, labeled as in (a), whereas (b) shows the second harmonic versus scan distance for voltages corresponding to the peaks in (c). SSHM clearly delineates the $\mathrm{n}^{+}$-doped, $\mathrm{p}$-doped and depletion regions. Regarding spatial resolution, the sharpest spatial feature corresponds to the $\mathrm{n}^{+} / \mathrm{p}$ depletion transition that takes place over $100 \mathrm{~nm}$. This, however, represents a limit that is due to the intermixing of the $n^{+} / p$ interface during the anneal and does not reflect the resolution limit of the microscope.

3.2 LANGMUiR-Blodgett Films. - A constant TH image of a 12-layer-thick LB film is shown in figure 5. From this image, it is clear that topographic information can be inferred from SSHM investigations of dielectrics. In particular, in the topographic line scan shown in figure 5b, steps corresponding to one or two bilayers as well as localized defects in the layer are easily identified.
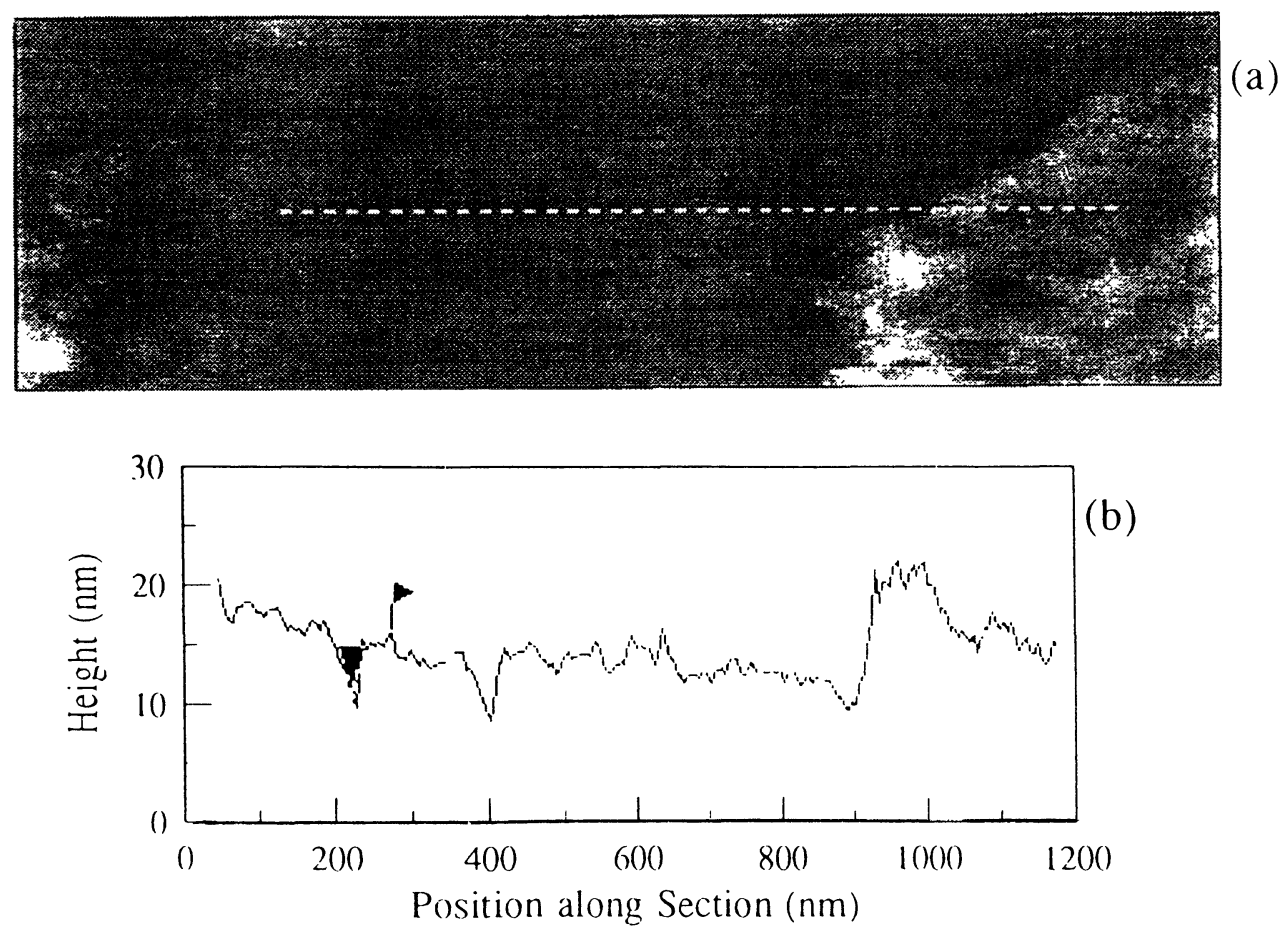

Fig. 5. - Constant TH image of a 12-layer-thick docosanoic acid LB film. Feedback conditions: dc bias $-0.5 \mathrm{~V}$ (tip voltage), ac power input $2 \mathrm{dBm}, 60 \mathrm{nV}$ TH signal, and $75 \%$ relative humidity. b) Section along the line shown in a). 


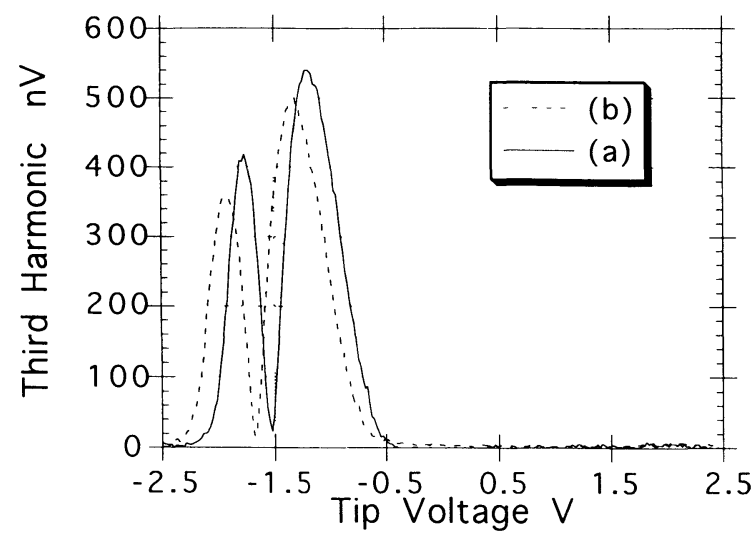

Fig. 6. - TH $(V)$ spectrum recorded a) on the LB film and b) on the substrate after removing the film. Feedback conditions: dc bias $-0.5 \mathrm{~V}$ (tip voltage), ac power input $2 \mathrm{dBm}, 10 \mathrm{nV} \mathrm{TH}$ signal, and $75 \%$ relative humidity.

Features of this type are not due to the underlying silicon substrate, because we do not observe them with bare substrates.

In order to understand the origin of the contrast in these images of LB films, TH $(V)$ spectra have been recorded. They are displayed in figure 6 for the LB film and the bare silicon substrate after removing the film with trichloroethane. The curves are very similar, meaning that there is no dominant contribution of the LB film to the nonlinearity of the tip-sample junction. In other words, the LB film behaves like a linear dielectric, and the contrast is due only to a modulation of the TH signal from the silicon substrate. This can be explained as follows. To a first approximation, the tip-sample junction is equivalent to a bilayer with one layer of air (water) of permittivity $\epsilon_{\mathrm{r}}=1\left(\epsilon_{\mathrm{r}}=80\right)$, and one layer of organic matter of permittivity $\epsilon_{\mathrm{r}} \approx 2.5$ [10]. For a given dc bias voltage applied to the tip, a thickness change in the LB layer results in a change in the total permittivity of the tip-sample gap and thus of the actual potential at the silicon surface. Consequently, there is a shift of the $C(V)$ characteristic and of the TH $(V)$ spectrum, which is converted into a change in the height of the piezo by the feedback loop. Note that a local change of the dielectric properties of a film of constant thickness would have the same effect. However, we do not think that this is the present case, because atomic force microscopy investigations of these films have produced comparable topographies (Fig. 7a). More precisely, steps one or more bilayers thick are frequently observed in LB films of cadmium or barium fatty-acid salts [15-17]. Occasionally in highly perturbated areas of the films (e.g. meniscus area and sample edges), steps can also be observed in LB films of fatty acids (Fig. 7b). Finally, the localized defects of the LB films allow the resolution of the SSHM to be estimated. For higher resolution, a higher constant TH reference level was used (typically a few $100 \mathrm{nV}$ ), and the relative humidity was decreased down to a few percent in order to minimize the size of the water nano-droplet in the tip-sample gap. Under these conditions, the smallest feature that could be resolved was $5 \mathrm{~nm}$. It should be noted that under wet conditions and for a constant TH signal of a few nanovolts, the tip-sample separation can be as large as $200 \mathrm{~nm}$, as measured in $\operatorname{TH}(z)$ experiments. Such conditions allow the investigation of soft films with very weak interactions between tip and sample and, thus, no degradation of the film during scanning. Of course this is only possible at the expense of resolution, which then drops to about $200 \mathrm{~nm}$. This large variation in the strength of the tip-sample interaction can be used to store information in an LB film. The write process corresponds to the local modification 


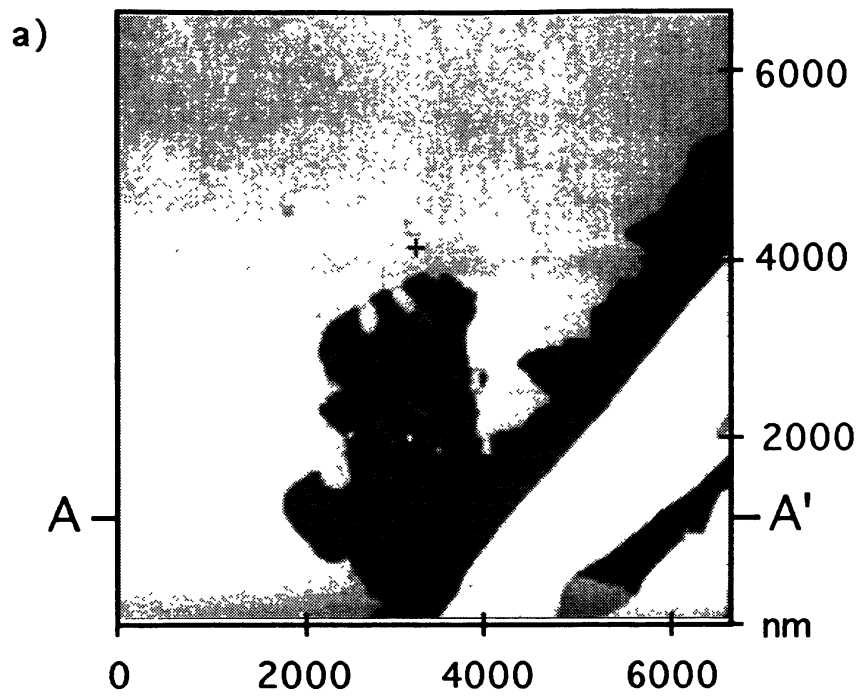

b)

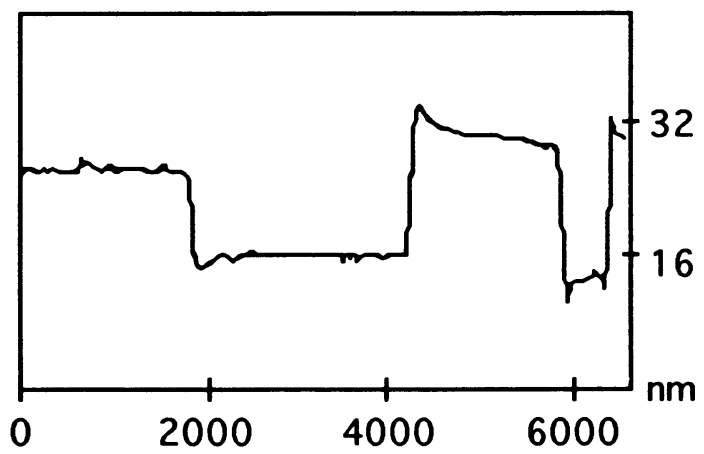

Fig. 7. - AFM image of a 12-layer-thick docosanoic acid LB film taken in constant height mode under ambient conditions using a silicon nitride tip on a cantilever with a spring constant of $0.38 \mathrm{~N} / \mathrm{m}$. b) Section along line $\mathrm{A}-\mathrm{A}^{\prime}$ shown in a).

of the film under strong interaction conditions; the read process is done by scanning under weak interaction conditions.

\section{Conclusion.}

SSHM investigations of semiconductors are sensitive to the $C / V$ characteristics by detecting higher harmonics resulting from nonlinearities in the tip-sample capacitance with voltage. Thus it can be used as a dopant profiler. It has been used to investigate LB films of docosanoic acid deposited on silicon. In this case, the film behaves like a linear dielectric, and the origin of the contrast is a modulation of the signal from the underlying silicon substrate, very likely because of thickness changes in the film. At present, the lateral topographic resolution of the SSHM on Langmuir-Blodgett films on silicon is $5 \mathrm{~nm}$. Finally, it should be noted that, although used to investigate an LB film insulator in this instance, the SSHM can be used to study other thin insulators on silicon, such as silicon dioxide or silicon nitride films. 


\section{Acknowledgments.}

We thank M.P. O'Boyle for doping gratings, D. Huber for silicon samples and acknowledge W. Knop and H. Benedikter for helpful discussions. B.M. acknowledges partial funding by the BBW Schweiz within the ESPRIT basic research project PRONANO (8523).

\section{References}

[1] Kochanski G.P., Phys. Rev. Lett. 62 (1989) 2285.

[2] Michel B., Mizutani W., Schierle R., Jarosch A., Knop W., Benedikter H., Bächtold W. and Rohrer H., Rev. Sci. Instrum. 63 (1992) 4080.

[3] Seifert W., Gerner E., Stachel M. and Dransfeld K., Ultramicroscopy 42-44 (1992) 379.

[4] Stranick S.J. and Weiss P.S., Rev. Sci. Instrum. 64 (1993) 1232.

[5] Mizutani W. and Michel B., unpublished.

[6] Fenner D.B., Biegelsen D.K. and Bringans R.D., J. Appl. Phys. 66 (1989) 419.

[7] Nakagawa Y., Ishitani A., Takahagi T., Kuroda H., Tokumoto H., Ono M. and Kajimura K., J. Vac. Sci. Technol. A8 (1990) 262.

[8] “Langmuir-Blodgett Films”, G.G. Roberts Eds. (Plenum Press, New York, 1990).

[9] Available from Material Analytical Services, Rayleigh NC 27604.

[10] See for example Nicollian E.H. and Brews J.R., "MOS Physics and Technology" (Wiley, New York, 1982).

[11] The absolute value comes from the lack of phase sensitivity in the setup.

[12] Johnson M.B. and Halbout J.M., J. Vac. Sci. Technol. B9 (1991) 508.

[13] In comparing the higher harmonics vs. bias voltage curves obtained for the lowest relative humidity conditions to those obtained for the highest relative humidity conditions, one merely notes a considerable difference of amplitude. As a consequence, on silicon surfaces, water seems to induce no noticeable higher harmonics generation because of its nonlinear permitivity. However, even for the lowest possible relative humidity conditions, water molecules are very likely to be adsorbed on the surface. They may play a role as a contaminant and account in part for the smaller feature observed at bias voltages corresponding to carrier population inversion at the semiconductor surface.

[14] Bourgoin J.-P., Johnson M.B. and Michel B., Appl. Phys. Lett. 65 (1994) 2045.

[15] Chi L.F., Eng L.M., Graf K. and Fuchs H., Langmuir 8 (1992) 2255.

[16] Meyer E., Overney R., Brodbeck D., Howald L., Lüthi R., Frommer J. and Güntherodt H.J., Phys. Rev. Lett. 69 (1992) 1777.

[17] Schwartz D.K., Wiswanathan R. and Zazadzinski J.A.N., Langmuir 9 (1993) 1384. 